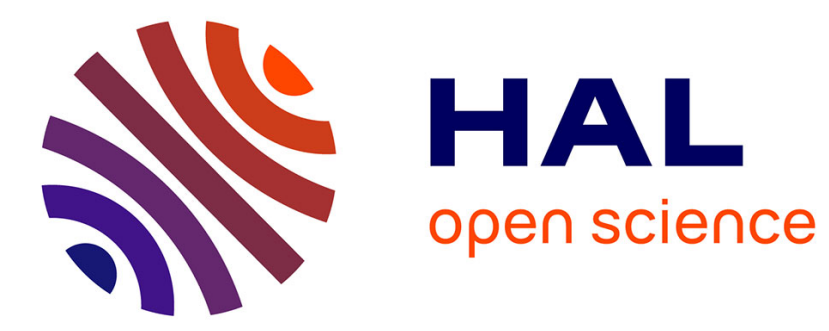

\title{
Géographie du mouvement, géographie en mouvement. La mobilité comme dimension du terrain dans l'étude des migrations
}

\author{
Julien Brachet
}

\section{- To cite this version:}

Julien Brachet. Géographie du mouvement, géographie en mouvement. La mobilité comme dimension du terrain dans l'étude des migrations: Communication au colloque " À travers l'espace de la méthode : les dimensions du terrain en géographie ", Arras, 18-20 juin 2008.. A travers l'espace de la méthode: les dimensions du terrain en géographie, Jun 2008, Arras, France. halshs-00358932v2

\section{HAL Id: halshs-00358932 \\ https://shs.hal.science/halshs-00358932v2}

Submitted on 28 Apr 2009

HAL is a multi-disciplinary open access archive for the deposit and dissemination of scientific research documents, whether they are published or not. The documents may come from teaching and research institutions in France or abroad, or from public or private research centers.
L'archive ouverte pluridisciplinaire HAL, est destinée au dépôt et à la diffusion de documents scientifiques de niveau recherche, publiés ou non, émanant des établissements d'enseignement et de recherche français ou étrangers, des laboratoires publics ou privés. 


\title{
Géographie du mouvement, géographie en mouvement. La mobilité comme dimension du terrain dans l'étude des migrations
}

\author{
Julien Brachet ${ }^{1}$ \\ Communication au colloque « À travers l'espace de la méthode : les dimensions du terrain en \\ géographie », Arras, 18-20 juin 2008
}

L'appel à la réflexivité, dans le but de dévoiler tant que possible les processus de connaissance, les opérations et les méthodes de production de la pensée scientifique, a concerné la plupart des disciplines, Prenant tantôt la forme d'une "phénoménologie de la phénoménologie » (Merleau-Ponty), d'une «ethnographie de l'ethnographie » (Clifford), d'une «sociologie de la sociologie » voire d'une "science de la science » (Bourdieu). Ce vaste -et relativement lent- tournant réflexif a fait émerger le «terrain» des sciences humaines et sociales en tant qu'objet de recherche à part entière. Longtemps considéré comme une simple évidence, notamment dans la littérature francophone et cela malgré l'existence de travaux précurseurs qui ont insistés sur le rôle des situations sociales dans l'analyse des objets ${ }^{2}$, le «terrain » est dorénavant systématiquement analysé et critiqué afin de prendre en compte les conditions subjectives et sociales de productions des savoirs (Ghasarian, 2002). L'objectivation des pratiques de terrain doit en effet permettre de révéler certains des biais inhérents à l'activité même de recherche empirique ${ }^{3}$, tant que possible de les rectifier et donc d'affiner la connaissance de l'objet. Ce qui accroît d'autant la rigueur de la démarche scientifique mise en œuvre (Leservoisier, 2005b). Néanmoins, où que l'on se positionne dans le débat sur la postmodernité et le constructivisme dans les sciences sociales, la réflexion sur les pratiques de terrain et la manière dont elles influent sur la nature des connaissances produites conduit généralement à un retour critique sur les choix méthodologiques, sur l'utilisation des outils ou sur sa propre subjectivité dans les moments formalisés de production de données. Ces tentatives d'objectivation des pratiques de terrain sont essentielles, mais elles traitent principalement des mécanismes de production et d'interprétation -et des régimes de scientificité- des données les plus visibles et qui posent question avec le plus d'évidence. Sans entreprendre une "géographie de la géographie », nous voudrions ici interroger un autre aspect des pratiques de terrain, des conditions de présence des chercheurs sur le terrain, spécifiquement dans le champ de la production des savoirs géographiques sur les migrations internationales et les mobilités. Il s'agit des pratiques spatiales (et donc sociales) des chercheurs. Entre fixité permanente et déplacements réguliers, toutes les pratiques de terrain sont possibles, sans pour autant que l'on accorde un intérêt particulier à cette question de la mobilité. Si la fixité est généralement considérée comme le moyen privilégié d'un ancrage en profondeur dans le terrain, selon les objets et les questionnements, quelles peuvent être les intérêts de la mobilité ? Quelles sont les incidences de cet aspect de la méthodologie, de la pratique et de l'expérience du terrain sur l'approche

\footnotetext{
${ }^{1}$ IRD - Université Paris 1 Panthéon-Sorbonne.

${ }^{2}$ Voir notamment les travaux de Georges Balandier (1985 [1955]; 1992 [1957]), puis ceux de Pierre Bourdieu dès les années 1960.

${ }^{3}$ L'existence du «terrain » résultant de l'introduction d'un acteur particulier, le chercheur, dans un milieu naturel et un champ social -ou groupe d'acteur, ou société- particuliers.
} 
que l'on peut avoir de nos objets d'étude et sur la nature des données produites ? À partir de travaux de recherche sur les migrations (trans)sahariennes menés au Niger pendant deux ans (2003-2005) dans le cadre de la préparation d'une thèse de doctorat (Brachet, 2007), nous allons voir dans quelle mesure la mobilité peut constituer une dimension signifiante de l'expérience dans les études migratoires, donnant accès à une autre dimension du terrain en même temps qu'elle transforme la perception même que l'on en a.

\section{Le terrain entre sédentarité et mobilité, entre « profondeur » et « superficialité »}

Il y a de nombreuses façons de parcourir le monde, de le découvrir, de l'étudier. Explorateurs et chercheurs, savants et aventuriers (la démarcation entre les uns et les autres ayant pu être ténue voire inexistante) en ont fait l'expérience. Un bref retour sur l'évolution de leurs manières de faire sur le terrain, sur l'évolution des modalités de leurs expériences empiriques montre notamment qu'entre sédentarité et mobilité d'un côté et profondeur et superficialité de l'autre, les concordances ne sont pas immuables. Ce qui invite à réinterroger les manières dont sont pensées et évaluées aujourd'hui, dans les communautés scientifiques, les pratiques de terrain.

\section{Explorations et explorateurs mobiles}

Aux XVIII ${ }^{\grave{e}}$ et XIX $^{\grave{e}}$ siècles, les descriptions de l'Afrique, et notamment de l'Afrique de l'Ouest et du Sahara, sont faites par des voyageurs mobiles, par des explorateurs qui circulent et «traversent » des régions entières du continent. Parmi les plus célèbres, on peut citer Mungo Park, qui voyage entre les actuels Sénégal et Nigeria à deux reprises, de 1795 à 1797 puis de 1805 à 1806 ; René Caillé rallie Saint-Louis du Sénégal à Tombouctou en 1828 ; Heinrich Barth qui va de Tripoli à Tombouctou puis qui revient par le lac Tchad entre 1849 à 1854 ; ou encore Henri Duveyrier, Gordon Laing, Louis-Gustave Binger, et bien d'autres. De retour en Europe, la plupart de ces explorateurs publient leurs récits de voyage qui, dans l'ensemble, sont de véritables récits ethnographiques et géographiques (Barth, 1860; Caillé, 1996 [1830]; Duveyrier, 1864; Park, 1996 [1799]). Comme le rappelle Isabelle Surun "l'exploration de l'Afrique au XIX è siècle peut être reconnue comme une entreprise géographique [qui] dépasse, et de loin, les visées des géographes eux-mêmes. [...] elle illustre le passage d'une géographie de la localisation et de l'inventaire à une géographie tournée vers l'homme» (Surun, 1998 : 37). L'une des dernières grandes expéditions mobiles d'exploration et de description scientifique de l'Afrique fut l'expédition Dakar-Djibouti, menée entre mai 1931 et février 1933 par Marcel Griaule (et dont Michel Leiris tira un magnifique carnet de voyage aux accents de journal intime : L'Afrique fantôme).

Le point commun de tous ces «explorateurs-scientifiques » est d'avoir privilégié les situations de voyage à celles de résidence. Qu'ils circulent seuls ou en groupe (en se joignant à des caravanes de commerçants, en participant aux colonnes militaires de la conquête coloniale, ou en organisant leurs propres expéditions), ils voyagent, ils se déplacent. Leurs découvertes, leurs observations, leurs relevés se font au cours du voyage, en passant ou tout au moins dans une logique de passage, ce qui n'empêche évidemment pas les étapes (volontaires ou contraintes) pouvant atteindre plusieurs mois parfois, ou les retours dans des zones déjà traversées (Spittler, 1996).

Du voyage au séjour : l'invention du terrain fixe 
Le véritable tournant a lieu au début du $X X^{\grave{e}}$ siècle, avec l'invention du terrain ethnographique fixe. Plusieurs auteurs ont montré combien la fameuse photo de la tente de Bronislaw Malinowski plantée au milieu du village des Iles Trobriands (lors de son séjour en 1914-1915) a fondé l'image classique du terrain en ethnologie (Clifford, 1992; Hannerz, 2003) ainsi qu'en géographie « humaine ». Pourtant, si l'on considère généralement que Malinovski est le principal inventeur du travail de terrain ethnologique moderne, dont la méthode privilégiée est l'observation participante de longue durée (Malinowski, 1963 [1922]), il serait plus juste de préciser que ce qu'il contribue à inventer, ce n'est pas le travail de terrain, mais le travail de terrain en site fixe. Car les explorateurs mobiles du XIXè siècle étaient déjà des géographes et anthropologues de terrain, apprenant les langues locales et pratiquant l'observation participante. Malinovski marque néanmoins une double rupture, à la fois avec les explorateurs du siècle précédent qui étaient davantage mobiles que sédentaires, et avec la pratique antérieure de l'ethnologie, qui consistait à travailler à partir de données recueillies par d'autres (Leservoisier, 2005a). ${ }^{4}$

L'anthropologie classique s'est ainsi développée en considérant que ses objets (la société, la culture, etc.) étaient marqués par et dans des espaces bien déterminés et délimités, qu'ils étaient supports et reflets de territoires «clos». Les normes qui déterminent les types d'expérience (durée, lieu) et d'interactions qui allaient constituer le terrain ont ainsi été influencées par ces représentations : un bon terrain devaient correspondre à un séjour de longue durée en un même lieu, au sein d'une même population dont le chercheur maîtrise la langue vernaculaire. L'idée de terrain fut alors liée à celle de "profondeur », et le terroir, le village, la ville, ont constitué des terrains par excellence, concentrés, stationnaires. L'unité de lieu permettant d'ancrer en profondeur ses recherches, son observation, avant éventuellement de l'élargir à un espace plus vaste. La mise en œuvre des enquêtes ethnographiques et la pratique classique du terrain étaient donc foncièrement locales, délimitées et sédentaires. Dans leurs modes de production des données empiriques, les anthropologues et les géographes ont ainsi longtemps privilégiés les situations de résidence ("dwelling") aux situations de voyage ("travel") (Clifford, 1997a).

Dans les études migratoires, cette conception du terrain s'est traduite par une focalisation des travaux sur les espaces de départ des migrants et sur les communautés d'immigrants installés dans leur pays d'accueil. Mais si la mobilité sur le terrain fut longtemps considérée comme étant une marque de «superficialité » s'opposant à l'ancrage « en profondeur» dans un terrain caractérisé par son unité de lieu, et impliquant la sédentarité, cette vision normative de l'expérience de terrain tend à changer, à s'ouvrir en fonction des objets étudiés et des questionnements (Clifford, 1997b; Marcus, 1998; Peraldi, 2002; Tarrius, 2002)

\section{Terrain et mobilité dans l'étude des migrations}

Lorsque l'objet étudié est caractérisé par la mobilité des acteurs à travers un espace très vaste, comme c'est le cas pour les mouvements migratoires au Sahara central, la première

\footnotetext{
${ }^{4}$ Quelques ethnologues avaient cependant déjà effectué des recherches empiriques (cf. Lewis H. Morgan, Franz Boas).
} 
question qui se pose est : quel(s) terrain(s) déterminer, délimiter ou construire ${ }^{5}$ pour étudier ces migrations de transit, et comment « pratiquer » ce terrain ?

\section{Carte 1 : Principaux flux migratoires à travers le Niger}

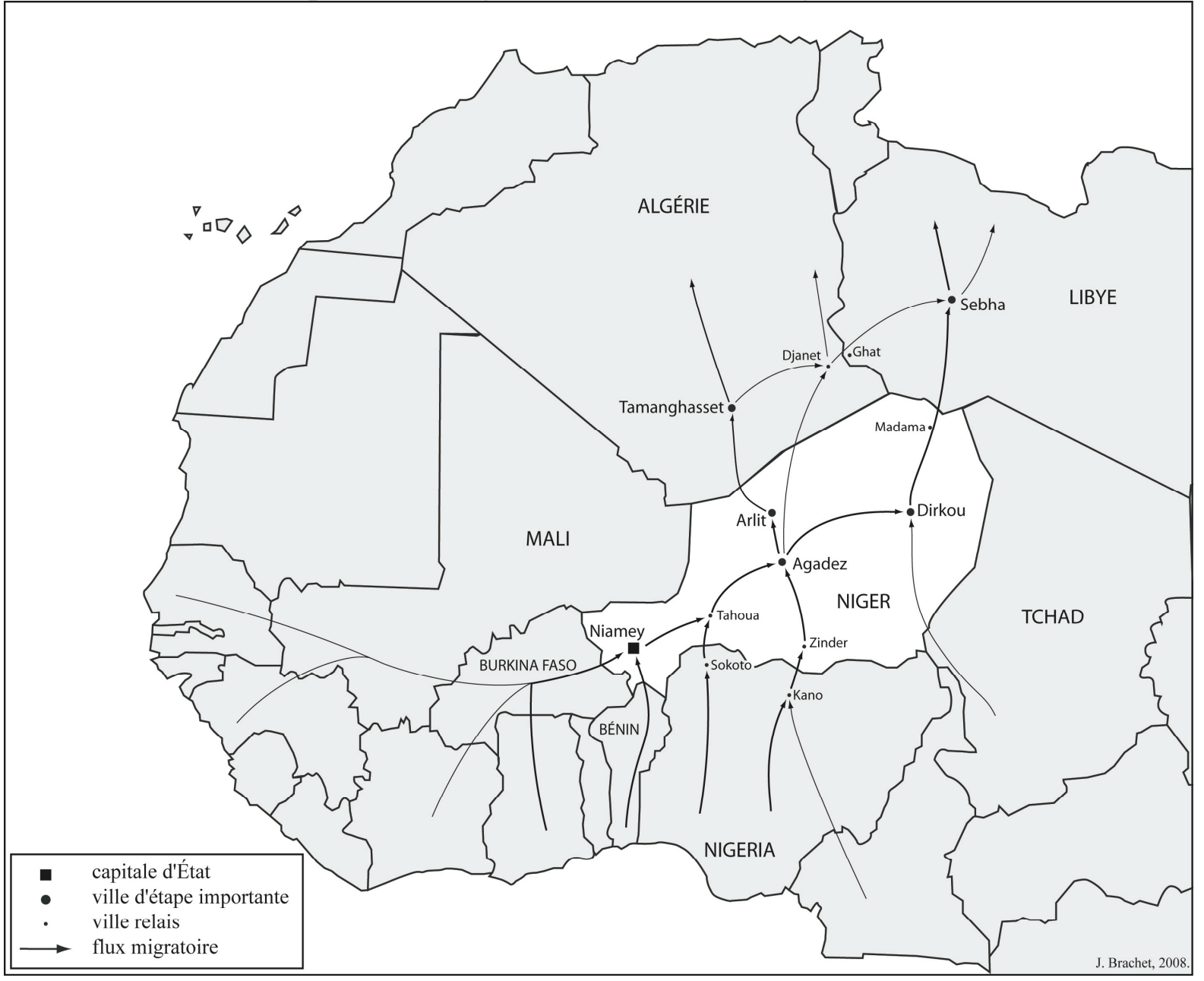

Pour y répondre, il faut repartir de considérations théoriques relatives à la manière dont on envisage la migration, le phénomène migratoire. Longtemps, la migration a été pensée à partir des espaces de départ et d'arrivée des migrants. Les études migratoires se basaient donc sur la description de lieux fixe, de moments fixe, pour parler de ces phénomènes. Or, justement, l'idée est d'envisager la migration comme un processus, comme fait social qui est caractérisé par la notion de «mouvement », et de réfléchir à ce que cela peut impliquer au niveau méthodologique.

Si la migration est avant tout mouvement, déplacement, ce qui implique le changement permanent des contextes dans lesquels se trouvent les migrants, alors son étude doit postuler l'instabilité des formes sociales qu'elle engendre et se centrer sur la (re)négociation permanente des relations entre les individus, sur l'adaptation des comportements, ou encore sur la redéfinition des projets des migrants. Et donc, partant de là, dans une optique de compréhension globale des parcours migratoires, des voyages des migrants pris en tant que combinaison d'étapes et de déplacements, on a intérêt à ne pas étudier les migrations

\footnotetext{
${ }^{5}$ Les anglophones parlent notamment de fieldwork ou field working, ce qui renvoie à l'idée que le terrain n'existe qu'à travers la présence du chercheur et l'action de recherche.
} 
uniquement à partir d'un endroit fixe, à partir d'un seul point d'observation, mais au contraire il apparaît nécessaire de faire varier les contextes d'observation et d'énonciation des acteurs. Ce qui suppose que l'observateur soit mobile. D'un point de vue méthodologique, la mobilité de l'observateur peut se décliner de deux manières : la mobilité sur le terrain et la «mobilité » comme terrain.

La mobilité sur le terrain. Du Sahara nigérien comme région d'étude à la détermination et à l'étude des sites de recherche

Dans le cas de la mobilité sur le terrain, une fois que la zone d'étude est choisie, à petite échelle, cela peut être un pays, une région voire même une ville, il faut ensuite déterminer les sites qui vont précisément constituer le terrain, ou au moins un de ces sites qui servira de point de départ. Dans mon cas, après avoir décidé de travailler sur les circulations au Niger, je me suis rendu dans le Nord du pays, dans la région saharienne d'Agadez dont la superficie est de $668000 \mathrm{~km}^{2}$ (cf. carte 2). Et c'est donc une fois sur place que j'ai pu déterminer plus précisément quels seraient les sites au sein desquels j'allais mener mes enquêtes et observations. Cette détermination du terrain a pris trois formes.

Dans un premier temps, $\mathrm{j}$ 'ai choisi de circuler et de travailler au hasard des endroits où les opportunités de déplacement me menaient, où j'avais «envie »d'aller. J'ai circulé ainsi pendant plusieurs mois, ce qui m'a permis de prendre contact avec ma zone d'étude de manière assez complète, plus exhaustive que si je m'étais concentré dès les premiers temps sur les endroits les plus « évidents », les plus «stratégiques ». L’intérêt de ce type de première approche est de ne pas trop se laisser diriger par ses représentations a priori, de se donner la possibilité de travailler ailleurs que là où on l'avait implicitement prévu, de laisser émerger de nouvelles pistes de recherche.

Ensuite, au gré de ces circulations dans la région d'Agadez, des rencontres et des discussions avec différents acteurs, j'ai pu commencer à déterminer un certain nombre de « micro-terrains », à me rendre dans des sites précis qui me semblaient plus importants que d'autres voire incontournables pour comprendre les phénomènes migratoires. Il s'agissait de certaines localités sahariennes, de puits d'étape où tous les acteurs du système migratoire se croisent, de lieux de transport où tout se négocie, ou encore de lieux de tensions, de violence et de négociation entre les pouvoirs publics et les acteurs privés, tels que les postes frontières, les barrières d'entrée et de sortie de villes, les lieux de chargements des véhicules clandestins.

Le troisième élément qui a participé de la détermination des sites qui allaient constituer mon terrain de recherche a été la contrainte. Certains endroits potentiellement très intéressants n'étaient pas accessibles, ou ne m'étaient pas accessibles. Je m'en suis rendu compte progressivement, au cas par cas. Le Nord-Est nigérien (au-delà de Ségédine) m'était « interdit » d'accès par les militaires de la zone, et je n'ai jamais pu me rendre dans les régions méridionales de la Libye ni de l'Algérie, faute de visa... Mais aucun motif officiel n'a jamais justifié l'inaccessibilité de ces terrains.

Finalement, ces trois facteurs que sont les hasards-opportunités, les choix volontaires et les contraintes extérieures, ont déterminé ma mobilité dans le Nord du Niger et dans le même temps les sites qui allaient constituer le terrain de mes recherches (carte 2). ${ }^{6}$

\footnotetext{
${ }^{6}$ Ces trois éléments de détermination du terrain sont ici présentés successivement, bien qu'en réalité ils se soient chevauchés et alternés en permanence tout au long de mes séjours.
} 
Carte 2 : La mobilité sur le terrain : de multiples sites d'étude

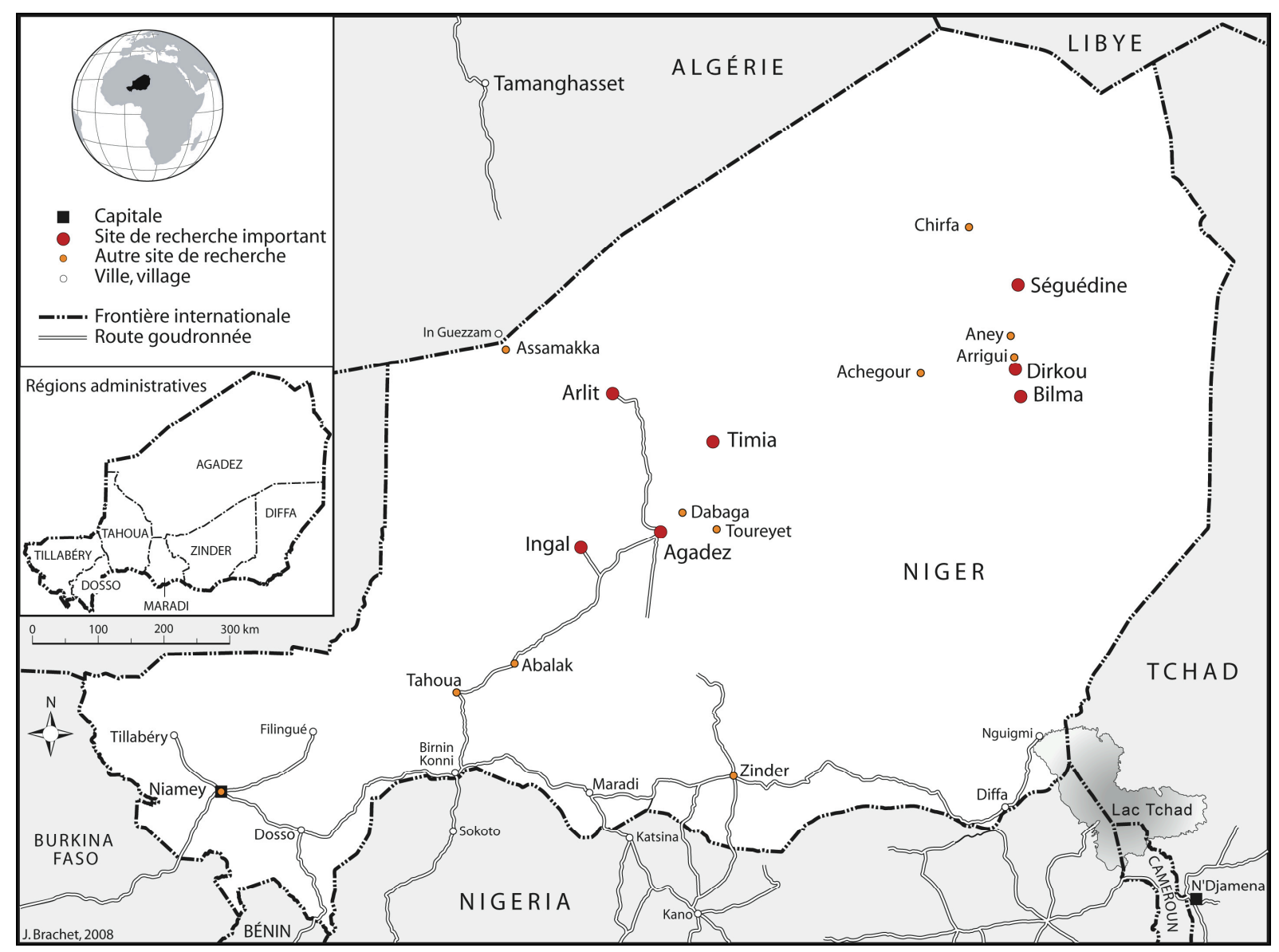

La mobilité sur le terrain, c'est-à-dire la circulation entre différents sites distincts, permet de mieux « contextualiser » chaque lieu d'enquête, qu'il s'agisse de villes, de villages ou d'oasis, et de s'intéresser à leurs relations, à leurs interactions, et finalement de mieux contrôler la production même de l'objet de recherche. D'une certaine manière, cela m'a permis de mener des enquêtes multi-situées à relativement grande échelle, au sens où les différents sites étaient directement reliés par voie terrestre les uns aux autres, et constituaient une sorte de réseaux ou de maillage.

Une approche similaire pourrait être envisagée avec des sites beaucoup plus éloignés les uns des autres, qui ne se succèdent pas directement dans les parcours migratoires, comme cela a été fait par exemple par Michel Peraldi ou Alain Tarrius au niveau de l'espace méditerranéen, et cela renverrait plus largement à la théorie de l'ethnographie multi-située développée par George Marcus (1995). Mais le fait que les sites soient distincts tout en étant relativement peu éloignés (d'une centaine à un millier de kilomètres) a un intérêt : faciliter les allers-retours, faciliter le fait de revenir à plusieurs reprises dans chaque lieu. Et chaque fois que je revenais dans un site où j'avais déjà travaillé, cela me permettait de porter un regard légèrement différent sur chaque phénomène observé, et d'obtenir de nouvelles informations lorsque que je recroisais une personne connue après un temps d'absence, et finalement, de mieux saisir les modes d'organisation des collectifs mis en co-présence par les mobilités (Berthomière, Hily, 2006). 
Ce mode d'approche du terrain, de pratique de la mobilité sur le terrain, permet d'étudier les transformations progressives :

- des discours des migrants concernant les raisons de leur départ (ces discours changent au fur et à mesure que les migrants s'éloignent de leurs pays de départ) ;

- de leur comportements économiques en fonction de leur appréhension de la suite du voyage ;

- de leurs sentiments d'appartenance, qui se transforment en fonction des situations rencontrées à chaque étape. Par exemple, l'appréhension croissante de la confrontation à l'extériorité (le désert) et à l'altérité (les agents de l'État), perçues comme de plus en plus dangereuses au fur et à mesure que les migrants pénètrent dans le Sahara, renforce dans le même temps un sentiments d'appartenance communautaire qui transcende les nationalités, les religions et les langues ;

- et aussi de leurs projets migratoires : les projet qui portent la migration ne sont pas fixes mais se recomposent en permanence, au fur et à mesure des parcours, selon les informations qui parviennent aux migrants, selon les opportunités qui se présentent à eux, et selon les contraintes rencontrées.

Tous ces aspects changeants des migrations (trans)sahariennes ne se révèlent et ne prennent sens que lorsqu'on travaille dans plusieurs étapes successives des parcours migratoires. Et il en va de même lorsqu'on s'intéresse aux politiques migratoires, dont les modalités d'application varient d'un endroit à l'autre : plus on s'éloigne des centres de décisions, plus on s'enfonce dans les confins sahariens, moins les agents de l'État se sentent contraints de remplir leurs fonctions officielles, et plus ils s'autorisent à agir en dehors du cadre de la légalité.

Ainsi, mener des enquêtes dans plusieurs sites permet de redonner de l'importance à la variation des contextes dans lesquels se trouvent les migrants au fur et à mesure des parcours. Ces changements de contexte, qui sont inhérents à la migration par étape, mettent en relief le fait que la migration doit être pensée comme un processus qui se réalise progressivement dans le temps et dans l'espace, et non comme un fait monolithique. Pour certains objets de recherche, tels que les migrations, le terrain multi-site offre ainsi de nombreux intérêts, voire de nouveaux horizons, par rapport au terrain fixe et unique. Ce qui ne veut pas dire que ce dernier n'a pas également son intérêt : le terrain multi-site ne remplace pas le terrain fixe, mais il le complète, il est différent et permet de produire des connaissances autres. L'intérêt de l'un et/ou l'autre dépend des questions que l'on pose.

Cependant, le terrain multi-site soulève quelques interrogations par rapport aux « normes » communément admises de la recherche empirique. Par exemple, plus il y a de sites, plus la durée de présence dans chaque site est réduite, ce qui peut faire resurgir l'opposition entre l'ancrage en profondeur et la superficialité, liée à la durée du terrain. Mais la légitimité du terrain multi-site, dans l'étude des migrations, passe par l'affirmation de son unité propre. C'est-à-dire que c'est bien l'ensemble des sites qui sont en relation les uns aux autres dans une structure réticulaire cohérente qui constituent le terrain, et non un seul ou plusieurs de ces sites pris isolément (Hannerz, 2003). Au final, cette forme de mobilité sur un terrain multi-site apparaît donc essentielle. Il reste néanmoins une limite à cette pratique : elle réduit la migration aux étapes fixes, elle fige ce qui est fluide. Or les parcours migratoires ne peuvent pas se réduire à une succession d'étapes fixes: entre chacune des étapes, les déplacements, les transports, constituent les liens entre les lieux et les moments du voyage. Ils font partie des espaces-temps de la migration. Au même titre que les étapes, ils constituent des situations potentielles d'enquête et d'observation, et doivent en cela être intégrés au terrain. 
Dans quelle mesure faut-il alors partager avec les acteurs l'expérience du mouvement afin d'en saisir les implications ? Quelles sont les possibilités et les enjeux d'une inscription de l'observateur dans la mobilité, afin d'appréhender la «mobilité » comme un terrain?

\section{La mobilité comme terrain. Du terrain multi-site au terrain réticulaire}

En travaillant entre les étapes des parcours, c'est-à-dire en et dans le mouvement, l'objectif est d'avoir accès à de nouvelles phases d'élaboration et de recomposition des projets des migrants, et de transformation de leurs sentiments d'appartenance selon les relations qui se nouent dans les moments de mobilité physique. Cette approche des phénomènes migratoires permet ainsi de les aborder réellement comme un processus continu, et non comme une succession d'étapes indépendantes.

Mais l'objectif d'un travail dans les flux migratoires est aussi d'avoir accès aux moments éphémères de contact entre les individus mobiles et les agents de l'État. Ces contacts discrets, formels ou informels, qui donnent parfois lieu à des tensions, ne sont accessibles et observables qu'en étant du côté des circulants, puisque les agents de l'État sont des agents de contrôles (police, gendarmerie, armée, douane) qui interviennent dans des endroits isolés le long des itinéraires sahariens (checkpoint) ou aux entrées et sorties des localités. Autant d'endroits où il n'est pas aisé d'être un observateur extérieur. Être dans les flux permet ainsi d'enquêter sur les situations de contrôle et de taxation, sur les pratiques illégales et parfois violentes des agents de l'État dans les confins sahariens du pays. Et dans le même temps, cela permet également d'observer les réactions des migrants et des transporteurs confrontés à ces situations, notamment le passage de la solidarité communautaire à des pratiques plus individualistes, ou les négociations entre individus, entre groupes, entre réseaux (Brachet, 2005, 2008).

Carte 3 : Des lieux et des itinéraires pour comprendre le mouvement : vers un terrain réticulaire 


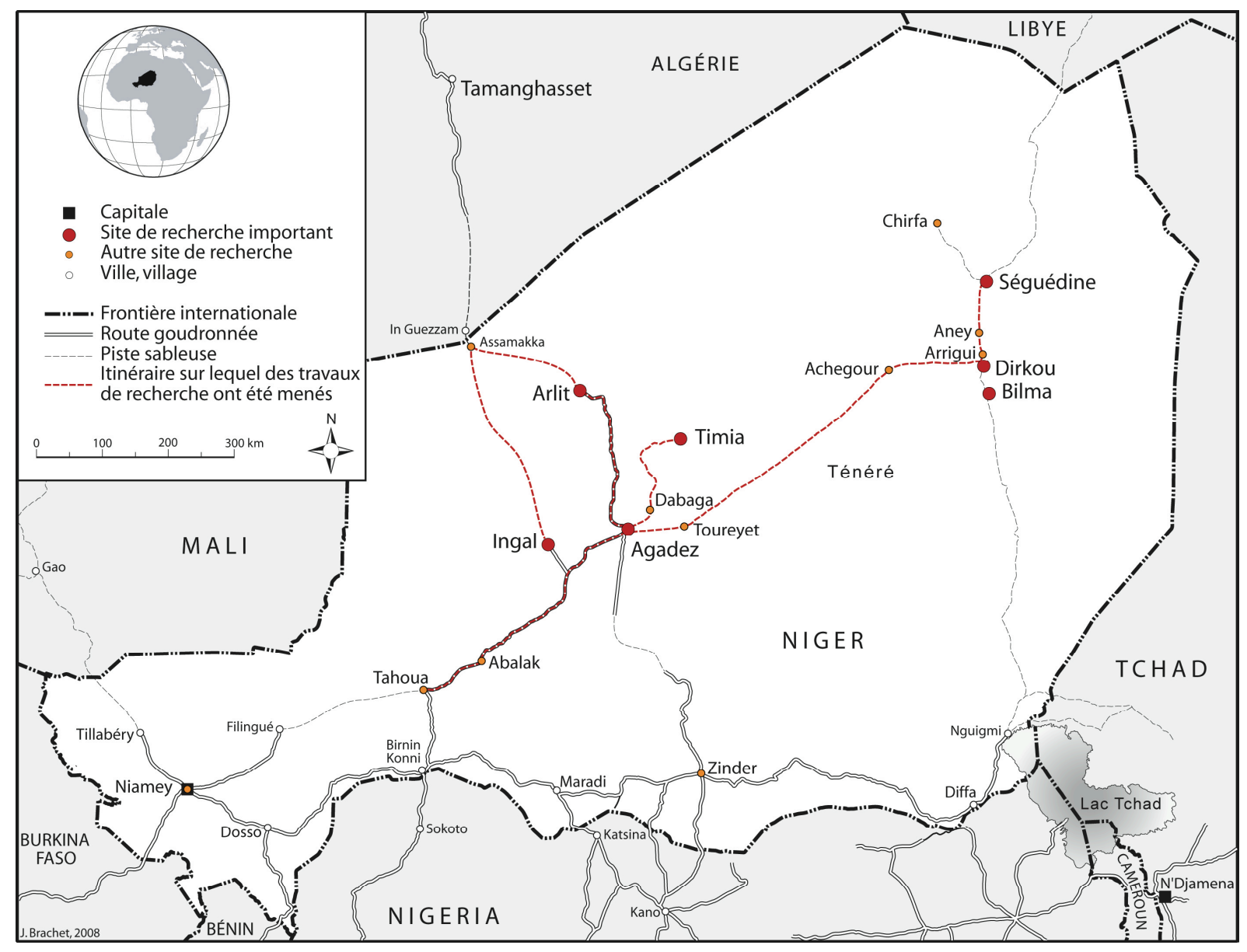

Pour réaliser une telle géographie des migrations (trans)sahariennes, pour avoir accès à ces lieux et à ces moments particuliers de la migration, il ne suffit plus de travailler dans les différents lieux d'étape, mais il faut également circuler entre ces lieux et faire de la mobilité un terrain. Il faut construire un «terrain réticulaire» en accordant a priori un potentiel heuristique identique aux points et aux lignes qui le constituent, c'est-à-dire aux localités et aux routes (carte 3). Or les routes empruntées, les pistes sahariennes, ne sont pas délimitées et peuvent varier d'un trajet à l'autre. Donc plus que les routes en elles-mêmes, plus que leur matérialité propre, ce sont les groupes de migrants qui se déplacent, et en se déplaçant qui font exister l'itinéraire et lui donnent sens, qui vont constituer le terrain. Donc d'une certaine manière on a un terrain qui est «mobile ». Et pour rester dans ce terrain, pour s'ancrer en profondeur dans un terrain ainsi construit, le chercheur doit donc également être mobile et circuler avec les migrants, et non pas à côté d'eux. En effet, plusieurs manières de circuler sur un terrain sont envisageables, allant de l'autonomie (qui représente des intérêts certains, en terme de confort de vie, de possibilité d'écriture, de gestion de son temps, etc.) à l'immersion au sein des populations dont on souhaite comprendre les logiques et les pratiques. La soumission volontaire à la dépendance logistique vis-à-vis de nos hôtes, qui de manière générale permet d'établir avec eux des relations répétées et prolongées, implique dans le domaine du transport de voyager avec et comme les migrants. Alors, et alors seulement, la « mobilité » peut être envisagée en tant que terrain spécifique de recherche. ${ }^{7}$

\footnotetext{
${ }^{7}$ Ce cas spécifique d'étude des migrations et des enjeux d'une mise en mouvement du chercheur sur le terrain invite à considérer l'ensemble des pratiques - et des conditions - matérielles du chercheur sur le terrain comme facteurs constitutifs de ce terrain, agissant sur le rapport à l'autre et aux lieux qui s'y instaure, et influant donc sur l'accès aux données et sur la nature des données produites.
} 
Concrètement, toute une partie de mes recherches sur les migrations (trans)sahariennes se sont déroulées dans les transports sahariens. Les pick-up et les camions de transport des migrants (fig. 1) étaient devenus des terrains d'observation privilégiés, notamment lors des traversées du Ténéré qui durent généralement 3 à 4 jours, et bien davantage lorsqu'il y a des pannes.

Figure 1: Des «terrains mobiles»: les camions de transport des migrants (Sahara nigérien)

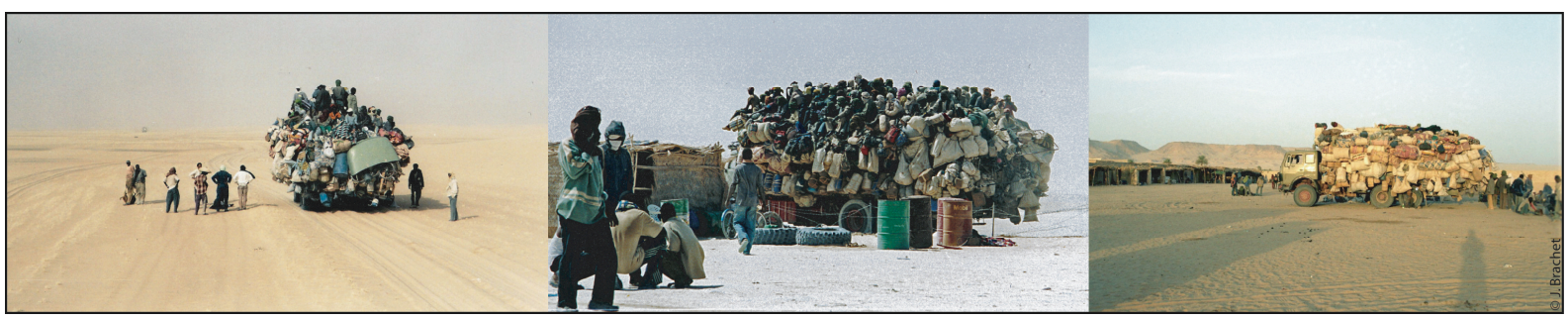

Finalement, le terrain de mes recherches sur les migrations (trans)sahariennes est constitué de plusieurs localités et des itinéraires qui les relient. Et c'est en intégrant à la fois des lieux fixes et des lieux mobiles qu'il trouve son unité. Si l'on revient à l'idée de « profondeur » nécessaire à la compréhension fine du terrain, on voit qu'une des dimensions des terrains d'étude des migrations est la mobilité, à la fois la mobilité sur le terrain et la mobilité comme terrain (les «terrains mobiles» constituant les liens entre les lieux fixes de la migration).

Comprendre le mouvement implique d'être immobile et d'observer ceux qui passent ; d'être mobile et d'observer ceux qui, immobiles, semblent passer ; d'être mobile et d'observer ceux qui, avec soi dans le mouvement, semblent immobiles. Ces trois approches de la migration, du mouvement dans la migration, visent à décrypter la tension entre mobilité et immobilité, entre voyage et installation temporaire, qui est fondatrice des processus migratoires. Il ne s'agit donc pas d'opposer ces «moments » l'un à l'autre mais bien de comprendre les relations qui les unissent comme les deux faces d'un même processus. La mobilité du chercheur sur le terrain, au lieu d'être synonyme de superficialité, devient ainsi l'impératif de son ancrage en profondeur. Et la géographie du mouvement devient une géographie en mouvement.

\section{Bibliographie}

BALANDIER Georges, 1985 [1955], Sociologie des Brazzavilles noires, Presses de la FNSP, Paris: 306.

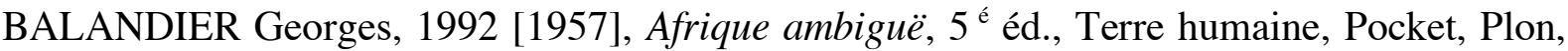
Paris: 293.

BARTH Heinrich, 1860, Voyages et découvertes dans l'Afrique septentrionale et centrale pendant les années 1849 à 1855, traduit de l'Allemand par Paul Ithier, tome I, Paris: 372 p. + ill.

BERTHOMIÈRE William, HILY Marie-Antoinette, 2006, "Décrire les migrations internationales. Les expériences de la co-présence", Revue Européenne des Migrations Internationales, 22 (2): 67-82.

BOYER Florence, 2003, "L'enfermement du voyage : construire des frontières pour passer la frontière", Cahiers d'Outre-Mer, 56 (222): 229-254. 
BRACHET Julien, 2005, "Migrants, transporteurs et agents de l'État : rencontre sur l'axe Agadez-Sebha", Autrepart, (36): 43-62.

BRACHET Julien, 2007, Un désert cosmopolite. Migrations de transit dans la région d'Agadez (Sahara nigérien), thèse de doctorat, département de géographie, Université Paris 1 Panthéon-Sorbonne, Paris: 459.

BRACHET Julien, 2008, D'une étape à l'autre. Transit et transport dans les migrations (trans)sahariennes, communication au colloque international African Migration Research: methods and methodology, International Migration Institute of Oxford University, 26-29 novembre 2008, Rabat (Maroc).

CAILLÉ René, 1996 [1830], Voyage à Tombouctou, La Découverte, Paris: 373.

CHANCEL Ausone de, DAUMAS Eugène, 1848, Le grand désert ou itinéraire d'une caravane du Sahara au pays des Nègres (royaume de Haoussas), Imprimerie et librairie centrales de Napoléon, Chaix et $C^{\text {ie }}$, Paris: 446.

CLIFFORD James, 1992, "Traveling Cultures", in Lawrence Grossberg, Cary Nelson, Paula Treichler (éds), Cultural Studies, Routledge, New York: 96-116.

CLIFFORD James, 1997a, Routes. Travel and Translation in the late twentieth century, Harward University Press, Cambridge-London: 408.

CLIFFORD James, 1997b, "Spatial Practices: Fieldwork, Travel, and the Disciplining of Anthropology", in Akhil Gupta, James Ferguson (éds), Anthropological Locations. Boundaries and Grounds of a Field Science, University of California Press, Berkeley: 185222.

DUVEYRIER Henri, 1864, Les Touareg du Nord, Editions Challamel Ainé, Paris: 502.

GHASARIAN Christian, 1997, "Les désarrois de l'ethnographe", L'Homme, 37 (143): 189198.

GHASARIAN Christian (éd.), 2002, De l'ethnographie à l'anthropologie réflexive. Nouveaux terrains, nouvelles pratiques, nouveaux enjeux, Armand Colin, Paris: 249.

HANNERZ Ulf, 2003, "Several Sites in One", in Thomas Hylland Eriksen (éd.), Globalisation: Studies in Anthropology, Pluto, London: 18-38.

HOURST Lieutenant E. A., 1898, La mission Hourst, Editions Plon, Paris: 482.

JEGANATHAN Pradeep, 2004, "Checkpoint. Anthropology, Identity, and the State", in Veena Das, Deborah Poole (éds), Anthropology in the Margins of the State, SAR Press, Sante Fe-Oxford: 67-80.

LESERVOISIER Olivier, 2005a, "L'anthropologie réflexive comme exigence méthodologique et épistémologique", in Olivier Leservoisier (éd.), Terrains ethnographiques et hiérarchies sociales. Retour réflexif sur la situation d'enquête, Karthala, Paris: 5-34.

LESERVOISIER Olivier (éd.), 2005b, Terrains ethnographiques et hiérarchies sociales. Retour réflexif sur la situation d'enquête, Karthala, Paris: 327.

LEVITT Peggy, GLICK SCHILLER Nina, 2004, "Conceptualizing simultaneity: A Transnational Social Field Perspective on Society", International Migration Review, 38 (3): 1002-10039.

MALINOWSKI Bronislaw, 1963 [1922], Les Argonautes du Pacifique occidental, Gallimard, Paris: 606.

MARCUS George E., 1995, "Ethnography in/of the World System: The Emergence of multisited Ethnography", Annual review of anthropology, 24: 95-117.

MARCUS George E., 1998, Ethnography through Thick and Thin, Princeton University Press, Princeton: 248.

MERLEAU-PONTY Maurice, 1999, Phénoménologie de la perception, Tel, Gallimard, Paris: 531 [ $1^{\text {ère }}$ éd. 1945]. 
MONTEIL Lieutenant-Colonel P.L., 1894, De Saint-Louis à Tripoli par le lac Tchad. Voyage au travers du Soudan et du Sahara accompli pendant les années 1890-91-92, Editions Félix Alcan, Paris: 404.

PARK Mungo, 1996 [1799], Voyage dans l'intérieur de l'Afrique, La Découverte, Paris: 354. PERALDI Michel (éd.), 2002, La fin des norias ? Réseaux migrants dans les économies marchandes en Méditerranée, Maisonneuve \& Larose, Paris: 495.

SCHIRMER H., 1898, Le dernier rapport d'un européen sur Ghât et les Touaregs de l'Aïr (journal de voyage d'Erwin de Bary, 1876-1877), Paris: 222.

SPITTLER Gerd, 1996, "Explorers in transit: Travels to Timbuktu and Agades in the nineteenth century", History and Anthropology, 9 (2-3): 231-253.

STJERNSTRÖM Olof, 2004, "Theory and migration. Towards a framework of migration and human actions", Cybergeo, Espace, Société, Territoire, (254): www.cybergeo.eu/index3827.html.

SURUN Isabelle, 1998, "L'exploration scientifique de l'Afrique au XIXème siècle est-elle une entreprise géographique ?" Finisterra. Revista portuguesa de geographia, XXXIII (65): 31-38.

TARRIUS Alain, 2002, La mondialisation par le bas. Les nouveaux nomades de l'économie souterraine, Balland, Paris: 170. 